\title{
Metabolic syndrome in polycystic ovary syndrome (PCOS): lower prevalence in southern Italy than in the USA and the influence of criteria for the diagnosis of PCOS
}

\author{
E Carmina, N Napoli, R A Longo, G B Rini and R A Lobo ${ }^{1}$ \\ Department of Clinical Medicine, University of Palermo, Palermo, Italy and ${ }^{1}$ Department of Obstetrics and Gynecology, Columbia University, New York, \\ New York, USA \\ (Correspondence should be addressed to R A Lobo; Email: ral35@columbia.edu)
}

\begin{abstract}
Objective: Metabolic syndrome (MBS) is a common disorder and is thought to be extremely prevalent in polycystic ovary syndrome (PCOS). In the USA the prevalence of MBS in PCOS has been reported to be as high as $43-46 \%$ using Adult Treatment Panel III (ATP-III) criteria. Because of differences in diet, lifestyle and genetic factors, we postulated that the prevalence of MBS might not be as high in Italian women. This study sought to determine the prevalence of MBS in Italian women using both the ATP-III and the World Health Organization (WHO) criteria and to determine whether the prevalence is influenced by the way in which PCOS is diagnosed.

Design: Assessment of the prevalence of MBS in 282 women with PCOS, aged 18-40 years, living in western Sicily. Eighty-five age- and weight-matched normal women served as controls.

Methods: Patients were divided into those with chronic anovulation and hyperandrogenism (classic PCOS; $n=225$ ) and others with hyperandrogenism and polycystic ovaries but who were ovulatory (ovulatory PCOS; $n=57$ ). A $75 \mathrm{~g}$ oral glucose tolerance test was carried out, as were lipid determinations; insulin resistance was assessed by the Quantitative Insulin-Sensitivity Check Index (QUICKI). We used ATP-III and WHO criteria to diagnose MBS.

Results: Using ATP-III criteria, the prevalence of MBS was 8.2\% and using WHO criteria it was $16 \%$ in Italian women with PCOS. In controls, the prevalence was $2.4 \%$ using both methods. In classic PCOS patients, MBS was higher ( $8.9 \%$ by ATP-III, $17.3 \%$ by WHO) than in ovulatory PCOS ( $5 \%$ and $10.6 \%$ respectively). Body weight significantly modified prevalence rates.

Conclusion: MBS is substantially higher in women with PCOS than in the general population, and the prevalence is higher in those women diagnosed by classic criteria. However, the prevalence of MBS in PCOS appears to be much lower in Italy than in the USA.
\end{abstract}

European Journal of Endocrinology 154 141-145

\section{Introduction}

Metabolic syndrome (MBS) which is a common disorder related to visceral obesity and insulin resistance (IR) is associated with atherosclerosis and cardiovascular (CV) disease $(1,2)$. The prevalence of MBS is high, occurring in $23.7 \%$ of the USA population over 20 years (3). The prevalence also increases with age, from $6.7 \%$ in the third decade to $43.5 \%$ in the 7 th decade $(4,5)$. The overall prevalence of MBS appears to be similar between the USA and European countries with reported rates of $23.5 \%$ in Spain and $23.9 \%$ in Portugal (6).

Several factors affect the prevalence of MBS. These include obesity (4), IR and diabetes (7), and polycystic ovary syndrome (PCOS) (8). In that $\mathrm{CV}$ risk factors are frequently present in women with $\operatorname{PCOS}(9,10)$, a recent suggestion was made to screen all obese women with PCOS for MBS $(11,12)$.
In attempting to determine the prevalence of MBS in PCOS, a major variable is the criteria used to define metabolic syndrome $(1,13)$. Most widely adapted are the criteria proposed by the American National Cholesterol Panel (Adult Treatment Panel III (ATP-III)) (14). These criteria require the presence of 3 of 5 common CV risk factors (increased waist circumference, blood pressure, elevated fasting blood glucose, low serum high-density lipoprotein (HDL)-cholesterol, and increased triglycerides). Using these criteria, the prevalence in PCOS has been reported to be extremely high, $43-46 \%(15,16)$, primarily on the basis of abnormal lipids and increased waist circumference.

However, in a recent paper we showed that in Italian women with PCOS, the prevalence of obesity and hypertriglyceridemia was markedly less than in USA women (17), and clearly constituents of the diet are different in the USA and Italy. Also, in the USA, alterations in 
HDL-cholesterol were linked to a higher proportion of saturated fat in the diet (17). In that diabetes and glucose intolerance are also less prevalent in Italian women compared with women in the USA with PCOS (18), we wished to determine the prevalence of MBS in Italian women with PCOS.

Although the prevalence of IR has been found to be similar between Italian and USA women with PCOS (10, 19), the prevalence of hypertriglyceridemia is far less in Italian women $(10,15)$. Thus the criteria used to diagnose MBS may alter the overall prevalence rate. It has been shown that the overall prevalence of MBS in Northern Italy is $34.1 \%$ using World Health Organisation (WHO) criteria (which stresses slightly different criteria) but is $17.8 \%$ using APT-III criteria (20).

Another potential variable in determining the true prevalence of MBS in PCOS is the criteria used for diagnosing PCOS. Using the clinical criteria of hyperandrogenism and chronic anovulation (often called National Institutes of Health (NIH) criteria (21)) a higher rate of $\mathrm{CV}$ risk factors may be found compared with women who were diagnosed by the ESHRE/ASRM criteria which place less emphasis on clinical findings (10).

Our purpose in this study was to determine the prevalence of MBS in Southern Italy compared with the reported rates in the USA. Further, we wished to determine if this prevalence is influenced by (a) the criteria used (ATP-III vs WHO) and (b) the way in which PCOS is diagnosed.

\section{Materials and methods}

\section{Subjects}

Two hundred and eighty-two women with PCOS were evaluated. We only included those women between the ages of 18 and 40 years who were living in western Sicily and who were of Italian ancestry. These women had been referred to the Department of Endocrinology and to the Department of Clinical Medicine of the University of Palermo between 1991 and 2004 for hyperandrogenism. They were diagnosed retrospectively according to the ESHRE/ASRM criteria (presence of at least two of the following symptoms: chronic anovulation, hyperandrogenism and polycystic ovaries) $(7,8)$. Normal circulating levels of 17-hydroxyprogesterone were used to exclude the diagnosis of non-classic 21-hydroxylase deficiency, while clinical data and/or urinary cortisol assessment were utilized to exclude the diagnosis of Cushing's syndrome. No patient had an androgen secreting tumor on the basis of finding very high androgen levels or abnormalities on computed tomography scan or magnetic resonance imaging. Some of these women had been included in other analyses (10). Anovulation was defined as serum progesterone $<10 \mathrm{nmol} / \mathrm{l}$. In patients who reported 'normal' menses, at least two consecutive cycles with low levels of serum progesterone were needed to make a diagnosis of anovulation. Menstrual cycles shorter than 25 days and longer than 34 days were considered abnormal.

Hyperandrogenism could be clinical (hirsutism, alopecia and/or acne) or subclinical, with only an increase in serum testosterone and/or dehydroepiandrosterone sulfate (21). Polycystic ovaries were diagnosed by pelvic or intravaginal sonography according to the Rotterdam conference criteria (22).

Patients with PCOS were divided into two subgroups according to their particular phenotype: 225 women had hyperandrogenism and chronic anovulation (classic (C)-PCOS) and 57 had hyperandrogenism and polycystic ovaries but normal ovulatory cycles (ovulatory (OV)-PCOS) (10). Eighty-five women of similar age (range 18-35 years) were randomly selected as normal controls. All subjects were sedentary and were not participating in any specific diet plan. Nevertheless, diets of all subjects were 'Mediterranean' in nature as described by us previously (17).

In all patients and in controls, body weight, waist circumference and blood pressure were measured. Fasting blood was obtained between 0800 and $1000 \mathrm{~h}$ for evaluation of glucose, insulin, HDL-cholesterol and triglycerides. Blood glucose was also determined during a $75 \mathrm{~g}$ oral glucose tolerance test with blood samples at 0 , 30, 60, 90 and $120 \mathrm{~min}$.

The prevalence of MBS was assessed by ATP-III (14) and by WHO criteria (20). According to ATP-III, MBS was diagnosed in subjects presenting with at least three out of the following criteria: increased waist circumference $(>88 \mathrm{~cm})$, low serum HDL-cholesterol $(<50 \mathrm{mg} / \mathrm{dl}$ in women $)$, increased serum triglycerides $(>150 \mathrm{mg} / \mathrm{dl})$, increased blood pressure $(>130 />85 \mathrm{~mm} \mathrm{Hg})$ and high fasting blood glucose $(>110 \mathrm{mg} / \mathrm{dl})$. Using WHO criteria, MBS was diagnosed in subjects presenting with one major criterion (diabetes, insulin resistance, altered glucose tolerance) plus two minor criteria (obesity (body mass index $(\mathrm{BMI})>30$ ), hypertension, dyslipidemia (assessed by HDL-cholesterol and triglycerides), microalbuminuria).

Insulin resistance was calculated by the Quantitative Insulin-Sensitivity Check Index (QUICKI) (23). Presence of diabetes and altered glucose tolerance were determined according to the American Diabetes Association criteria (24). Insulin was determined with a double antibody method using reagents obtained from Linco Research, Inc. (St Charles, MO, USA). Blood glucose was determined using the glucose oxidase method. HDL-cholesterol was determined using the cholesterol esterase method following selective precipitation of apolipoprotein-B containing lipoproteins with a polyanion solution. Triglycerides were determined enzymatically as glycerol on a Roche automated chemistry analyzer following hydrolysis with lipase. All lipid analyses had intra- and interassay variations of $<3 \%$.

Institutional Review Board approval was obtained, and all patients and controls gave written consent. 
Table 1 Clinical data in women with PCOS and in normal controls.

\begin{tabular}{lccccc}
\hline & $\begin{array}{c}\text { Age } \\
\text { (years) }\end{array}$ & BMI & Waist circumference & $\begin{array}{c}\text { Systolic blood pressure } \\
(\mathrm{mm} \mathrm{Hg})\end{array}$ & $\begin{array}{c}\text { Diastolic blood pressure } \\
(\mathrm{mm} \mathrm{Hg})\end{array}$ \\
\hline Normal control women & $25.2 \pm 0.2$ & $23.3 \pm 0.6$ & $85 \pm 0.1$ & $112 \pm 1.4$ & $74 \pm 1.1$ \\
PCOS & $24.9 \pm 0.1$ & $27.2 \pm 0.3^{*}$ & $89 \pm 0.1^{*}$ & $118 \pm 0.7^{*}$ & $81 \pm 0.7^{*}$ \\
\hline
\end{tabular}

BMI, body mass index. ${ }^{\star} P<0.01$ versus normal control women.

\section{Statistical analyses}

Analysis of variance was used for comparisons. Post hoc testing was carried out by Student's $t$-test with $\log$ transformation. Analysis of covariance was used to evaluate the role of BMI on metabolic parameters. $P<0.05$ was considered statistically significant. All data are expressed as means \pm standard error.

\section{Results}

The clinical data of women with PCOS and of controls are depicted in Table 1. Patients with PCOS had higher BMI, waist circumference and blood pressure compared with normal women $(P<0.01)$. Obesity $($ BMI $>30)$ was present in 73 women with PCOS $(25.9 \%)$ while 104 patients were overweight $(36.9 \%)$ and 105 patients were of normal weight $(37.2 \%)$. Increased waist circumference was found in 110 women (39\%) and increased blood pressure in 21 patients $(7.3 \%)$.

Women with PCOS had higher insulin and triglycerides and lower insulin-resistant indices and HDLcholesterol than normal controls of the same age (Table 2). Increased blood glucose was found in 9 women $(3.1 \%)$ while altered glucose tolerance was found in 35 women $(12.4 \%)$. Increased insulin $(>12.5 \mu \mathrm{U} / \mathrm{ml})$ was found in 147 women $(52.1 \%)$ and reduced insulin sensitivity was found in 172 women $(61 \%)$. Low HDL-cholesterol $(<50 \mathrm{mg} / \mathrm{dl})$ was found in 127 women $(45.1 \%)$ while only 26 women with PCOS $(9.3 \%)$ had increased triglycerides (>150 mg/dl) (Fig. 1).

Using ATP-III criteria, 23 women with PCOS had MBS, giving a prevalence of $8.2 \%$. Using WHO criteria, 45 women with PCOS had MBS, giving a prevalence of $16 \%$. The prevalence of MBS in the control population was $2.4 \%$ by both ATP-III and WHO criteria (Fig. 2).

The prevalence of MBS was higher in C-PCOS (8.9\% by ATP-III and $17.3 \%$ by WHO) compared with women with OV-PCOS ( $5 \%$ by ATP-III and $10.6 \%$ by WHO).
The influence of body weight on the diagnosis of MBS also was assessed. Among the women diagnosed by ATP-III criteria, 16 women were obese $(21.9 \%$ of the women with PCOS who were obese), while 5 were overweight $(4.8 \%)$ and only 2 were of normal weight $(1.9 \%)$. Among the women diagnosed by WHO criteria, 28 were obese (38.4\% of obese PCOS group), 14 were overweight (13.5\% of overweight PCOS patients) and 3 (2.9\% of normal weight PCOS) had normal weight. The two normal controls who had metabolic syndrome were both obese.

\section{Discussion}

Our data suggest that MBS in women with PCOS is less common in southern Italy compared with women in the USA. Using the most used method (the ATP-III criteria) to assess the presence of metabolic syndrome in our population, the prevalence of MBS was only $8.2 \%$ in comparison with a prevalence of $43-46 \%$ reported in the USA $(15,16)$. While all diagnostic criteria were less common in our patients than in USA patients $(15,16)$, the main difference was in the lower prevalence of hypertriglyceridemia (increased triglycerides were present in only $9.3 \%$ of patients).

In a previous study, we observed that while USA women (mostly from Pennsylvania) and Italian women have similar androgen levels, similar blood pressure, similar total cholesterol and similar low-density lipoprotein-cholesterol, USA women have higher body weight, higher fasting insulin, lower glucose:insulin ratio, lower HDL-cholesterol and higher triglycerides (17). Most of these differences occurred as a result of the higher prevalence of obesity in the USA groups, but other factors, including characteristics of the diet, may also be responsible. In our previous study, the quantity of saturated fat in the diets of USA women was almost double that of Italian women (17).

The difference in the prevalence of MBS is higher if WHO criteria are used. By using WHO criteria, the

Table 2 Metabolic data in women with PCOS and in normal controls.

\begin{tabular}{lcccc}
\hline & Insulin $(\mu \mathrm{U} / \mathrm{ml})$ & QUICKI & HDL-cholesterol $(\mathrm{mg} / \mathrm{dl})$ & Triglycerides $(\mathrm{mg} / \mathrm{dl})$ \\
\hline Normal control women & $9 \pm 2$ & $0.360 \pm 0.02$ & $51 \pm 10$ & $72 \pm 21$ \\
PCOS & $16.2 \pm 7^{\star *}$ & $0.324 \pm 0.02^{\star *}$ & $48 \pm 10^{\star}$ & $90 \pm 38^{\star *}$ \\
\hline
\end{tabular}

${ }^{\star} P<0.05,{ }^{* \star} P<0.01$ versus normal control women. 
144 E Carmina and others

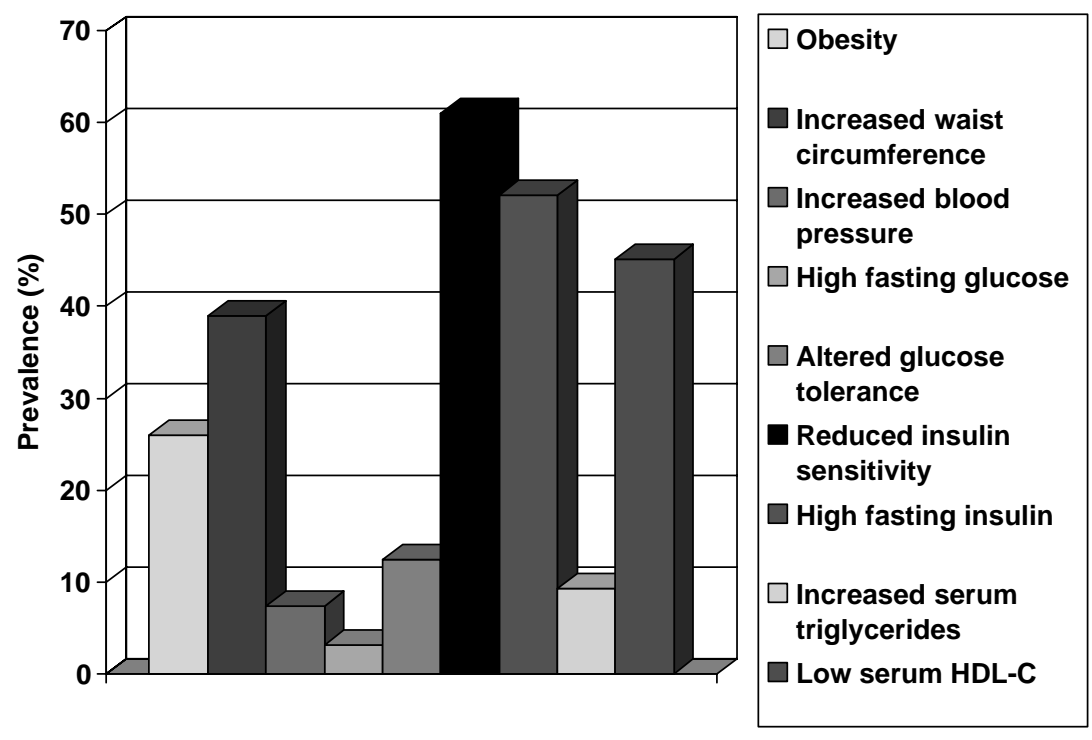

EUROPEAN JOURNAL OF ENDOCRINOLOGY (2006) 154

Figure 1 Prevalence of different elements of the metabolic syndrome in women with PCOS. prevalence of MBS doubled to $16 \%$ (still much lower than the data reported in USA women). While WHO criteria usually correlate with ATP-III criteria, in some populations differences have been reported $(5,20)$. This may be because WHO criteria are less dependent on obesity and lipid levels. It is our view that in the assessment of young women with PCOS, particularly in Italy where there is a lower prevalence of obesity and hypertriglyceridemia, WHO criteria would be preferred over the National Cholesterol Education Program ATP-III criteria as they are more discriminating.

We also wished to determine if the prevalence of MBS is influenced by diagnostic criteria for PCOS. In previous papers evaluating the prevalence of MBS in PCOS, PCOS was diagnosed according to NIH criteria $(15,16)$. Here, we have determined the prevalence of MBS using ESHRE/ASRM criteria as well. While MBS was more common in patients with C-PCOS (i.e. in patients diagnosed by $\mathrm{NIH}$ criteria) than in ovulatory women with PCOS with a focus on ultrasound findings, in women diagnosed as having C-PCOS, the prevalence is still much higher in the USA than in Italy.

As expected, MBS was more prevalent in obese patients with PCOS. However, overweight patients also had an increased prevalence of MBS. In a recent consensus conference, it was suggested that all obese women with PCOS should be screened for MBS (11, 12). We suggest that this should be extended to include merely overweight patients as well.

The prevalence of MBS in PCOS was 3.4-6.6 times higher than in the matched control population suggesting that PCOS per se results in a higher prevalence of MBS. Further, our data also suggest that the cardiovascular risk in our patients with PCOS is much higher than that suggested by the simple determination of MBS (using ATP-III criteria); a constellation of $\mathrm{CV}$ risk factors was increased in the majority of our patients with PCOS (10).

In conclusion, it is important to point out that the prevalence of MBS in PCOS is strongly influenced by the criteria used to diagnose metabolic syndrome as well as the criteria used to diagnose PCOS. However, it seems clear that MBS is much more common in PCOS than in the general population in women of similar age, and that women with PCOS who are diagnosed by classic criteria have a higher prevalence of MBS. Interestingly, the prevalence of MBS in PCOS is lower in Italian women than in women from the USA suggesting that genetic factors, but mostly differences in lifestyle and diet profoundly influence the prevalence of MBS in women with PCOS.

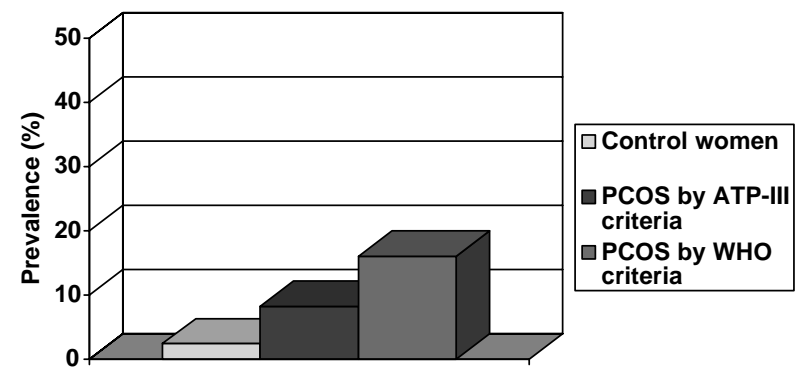

Figure 2 Prevalence of metabolic syndrome in control women and in women with PCOS according to the ATP-III and WHO criteria. 


\section{References}

1 Doelle GC. The clinical picture of metabolic syndrome. Postgraduate Medicine 2004116 35-38.

2 Isomaa B, Almgren P, Tuomi T, Forsen B, Lahti K, Nissen M, Taskinen MR \& Groop L. Cardiovascular morbidity and mortality associated with the metabolic syndrome. Diabetes Care 200124 $683-689$

3 Ford ES, Giles WH \& Dietz WH. Prevalence of the metabolic syndrome among US adults: findings from the third National Health and Nutrition Examination Survey. Journal of the American Medical Association 2002287 356-359.

4 Park YW, Zhu S, Palaniappan L, Heshka S, Carnethon MR \& Heymsfield SB. The metabolic syndrome: prevalence and associated risk factor findings in the US population from the third National Health and Nutrition Examination Survey, 19881994. Archives of Internal Medicine $2003163427-436$.

5 Alvarez Cosmea A, Lopez Hernandez V, Suarez Garcia S, Aria Garcia T, Preto Diaz MA \& Diaz Gonzalez L. Differences in the prevalence of metabolic syndrome according to the ATP-III and WHO definitions. Medical Clinics 2005124 368-370.

6 Santos AC, Lopes C \& Barros H. Prevalence of metabolic syndrome in the city of Porto. Revista Portuguesa de Cardiologia (Lisboa) 2004 23 45-52.

7 Seidell JC. Obesity, insulin resistance and diabetes - a worldwide epidemic. British Journal of Nutrition 200083 (Suppl. 1) 55-58.

8 Lobo RA \& Carmina E. The importance of diagnosing the polycystic ovary syndrome. Annals of Internal Medicine 2000132 989-993.

9 Talbott EO, Zborowski JV, Rager JR, Boudreaux MY, Edmundowicz DA \& Guzick DS. Evidence for an association between metabolic cardiovascular syndrome and coronary and aortic calcification among women with polycystic ovary syndrome. Journal of Clinical Endocrinology and Metabolism 200489 5454-5461.

10 Carmina E, Longo RA, Rini GB \& Lobo RA. Phenotypic variation in hyperandrogenic women influences the finding of abnormal metabolic and cardiovascular risk parameters. Journal of Clinical Endocrinology and Metabolism 200590 2545-2549.

11 Rotterdam ESHRE/ASRM-Sponsored PCOS Consensus Workshop Group, Revised 2003 consensus on diagnostic criteria and longterm health risks related to polycystic ovary syndrome. Fertility and Sterility $2004 \mathbf{8 1} 19-25$.

12 Rotterdam ESHRE/ASRM-Sponsored PCOS Consensus Workshop Group, Revised 2003 consensus on diagnostic criteria and longterm health risks related to polycystic ovary syndrome. Human Reproduction 200419 41-47.

13 Prabhakaran D \& Anand SS. The metabolic syndrome: an emerging risk state for cardiovascular disease. Vascular Medicine $2004955-68$.
14 Executive Summary of the Third Report of the National Cholesterol Education Program (NECP), Expert Panel on Detection, Evaluation and Treatment of High Blood Cholesterol in Adults (Adult Treatment Panel III). Journal of the American Medical Association $20012852486-2497$.

15 Glueck CJ, Papanna R, Wang P, Goldenberg N \& Sieve-Smith L. Incidence and treatment of metabolic syndrome in newly referred women with confirmed polycystic ovarian syndrome. Metabolism $200352908-915$.

16 Apridonidze T, Essah P, Iuorno M \& Nestler JE. Prevalence and characteristics of metabolic syndrome in women with polycystic ovary syndrome. Journal of Clinical Endocrinology and Metabolism $2005901929-1935$.

17 Carmina E, Legro R, Stamets K, Lowell J \& Lobo RA. Differences in body weight between American and Italian women with the polycystic ovary syndrome: influence of the diet. Human Reproduction $2003112289-2293$.

18 Gambineri A, Pelusi C, Manicardi E, Vicennati V, Cacciari M, Morselli-Labate AM, Fagotto U \& Pasquali R. Glucose intolerance in a large cohort of Mediterranean women with polycystic ovary syndrome. Phenotype and associated factors. Diabetes 200453 2353-2358.

19 Carmina E \& Lobo RA. Use of fasting blood to assess the prevalence of insulin resistance in women with polycystic ovary syndrome. Fertility and Sterility $2004 \mathbf{8 2} 661-665$.

20 Bonora E, Kiechl S, Willeit J, Oberhollenzer F, Egger G, Bonadonna RC \& Muggeo M. Metabolic syndrome: epidemiology and more extensive phenotypic description. Cross-sectional data from the Bruneck study. International Journal of Obesity-Related Metabolic Disorders 200327 1283-1289.

21 Carmina E. Prevalence of idiopathic hirsutism. European Journal of Endocrinology $1998139421-423$.

22 Balen AH, Laven JS, Tan SL \& Dewailly D. Ultrasound assessment of the polycystic ovary: international consensus definitions. Human Reproduction Update 20039 505-514.

23 Katz A, Nambi SS, Mather K, Baron AD, Follmann DA, Sullivan G \& Quon MJ. Quantitative Insulin-Sensitivity Check Index: a simple, accurate method for assessing insulin sensitivity in humans. Journal of Clinical Endocrinology and Metabolism 2000 $852402-2410$

24 Report of the Expert Committee on the Diagnosis and Classification of Diabetes Mellitus. Diabetes Care 199726 3160-3167.

Received 2 June 2005

Accepted 26 September 2005 